\title{
Avaliação de risco de úlcera por pressão: propriedades de medida da versão em português da escala de Waterlow*
}

\author{
Pressure ulcer risk assessment: sensitivity and specificity properties of the portuguese version of \\ Waterlow scale \\ Evaluación de riesgo de úlcera por decúbito: propiedades de medida de la escala de Waterlow en el \\ idioma portugués
}

\section{Alessandra Bongiovani Lima Rocha ${ }^{1}$, Sonia Maria Oliveira de Barros $^{2}$}

\begin{abstract}
RESUMO
Objetivo: Esta pesquisa teve como objetivo conhecer os índices de especificidade e sensibilidade da versão adaptada à língua portuguesa da escala de Waterlow. Métodos: Aplicação clínica da escala adaptada para avaliar prospectivamente 44 pacientes, por 15 dias consecutivos, em unidades clínicas de um hospital universitário de porte extra. Resultados: Os pacientes que desenvolveram úlcera por pressão apresentaram

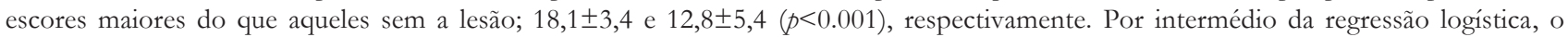
método Stepwise demonstrou que os escores para tipo de pele e apetite foram significantes para predizer o desenvolvimento de úlcera por pressão. A análise da curva ROC mostrou que o escore 15 obteve os melhores índices de sensibilidade e especificidade, 87\% e 76\%, respectivamente. Conclusão: Os resultados demonstraram que a escala de Waterlow adaptada à língua portuguesa foi um instrumento preciso e eficaz para predizer o desenvolvimento de úlceras por pressão na população do estudo.
\end{abstract}

Descritores: Úlcera de pressão; Medição de risco; Avaliação em enfermagem/métodos

\begin{abstract}
Objective: To evaluate the sensitivity and specificity properties of Portuguese version of the Waterlow scale. Methods: Field test of the scale among forty-four patients admitted to medical units in over sized university hospital for a period of 15 days. Results: Patients who developed pressure ulcer had higher scores on the Waterlow scale than those without PU (18.1 \pm 3.4 and $12.8 \pm 5.4$, $p<0.001$, respectively). Stepwise logistic regression indicated that skin type and appetite were significant predictors for the development of PU. The ROC curve analysis showed that score of 15 suggest the best combination of sensitivity and specificity, $87 \%$ and $76 \%$, respectively. Conclusion: The results suggest that the Portuguese version of the Waterlow scale was an accurate and effective tool to predict the development of pressure ulcer in patients admitted to medical surgical units.

keywords: Pressure ulcer; Risk assessment; Nursing assessment/methods
\end{abstract}

\section{RESUMEN}

Objetivo: Esta investigación tuvo como objetivo conocer los índices de especificidad y sensibilidad de la versión adaptada al idioma portugués de la escala de Waterlow. Métodos: Aplicación clínica de la escala adaptada para evaluar prospectivamente a 44 pacientes, por 15 días consecutivos, en unidades clínicas de un hospital universitario de extra porte. Resultados: Los pacientes que desarrollaron úlcera por decúbito presentaron escores mayores que aquellos sin la lesión; 18,1 $\pm 3,4$ y 12,8 $\pm 5,4(p<0.001)$, respectivamente. Por intermedio de la regresión logística, el método Stepwise demostró que los escores para el tipo de piel y apetito fueron significativos para predecir el desarrollo de úlcera por decúbito. El análisis de la curva ROC mostró que el escore 15 obtuvo los mejores índices de sensibilidad y especificidad, el 87\% y $76 \%$, respectivamente. Conclusión: Los resultados demostraron que la escala de Waterlow adaptada al idioma portugués fue un instrumento preciso y eficaz para predecir el desarrollo de úlceras por decúbito en la población del estudio.

Descriptores: Úlcera por decúbito; Medida del riesgo; Evaluación en enfermería/métodos

\footnotetext{
* Trabalho extraído da dissertação de mestrado, apresentada à Universidade Federal de São Paulo - UNIFESP - São Paulo (SP), Brasil.

1 Docente do Curso de Graduação em Enfermagem dos Centros Universitários São Camilo - CUSC e Nove de Julho - UNINOVE, São Paulo (SP), Brasil.

${ }^{2}$ Livre Docente na Saúde da Mulher; Professora Associado do Departamento de Enfermagem da Universidade Federal de São Paulo - UNIFESP - São Paulo (SP), Brasil.
} 


\section{INTRODUÇÃO}

O desenvolvimento de úlceras por pressão (UP) é um grave problema de enfermagem, pois, freqüentemente, é associado à má qualidade da assistência e exige uma grande demanda de tempo e dinheiro para tratamento das lesões, sobretudo quando a prevenção recebe menos atenção, quando não existem programas específicos voltados para esse problema.

$\mathrm{Na}$ escolha de um método de avaliação de risco para o desenvolvimento de UP, a eficácia e a facilidade de aplicação do instrumento de medida são consideradas. Os dois itens devem ser levados em conta, para que a equipe de enfermagem identifique corretamente, quais pacientes estão em perigo de desenvolver a lesão, evitando o uso inadequado de medidas preventivas.

Vários métodos de avaliação de risco para UP têm sido desenvolvidos, seguindo o pioneiro estudo que apresentou a escala de Norton em 1962, que por meio da análise e pontuação dos fatores de risco predisponentes ao desenvolvimento da úlcera obtém um escore com o objetivo de implementar medidas preventivas precisas, conforme o grau de risco de cada paciente $^{(1)}$.

Atualmente, há em torno de 40 escalas de avaliação de risco e seus estudos, na maioria revisões da literatura, são opiniões de especialistas ou adaptações de instrumentos já existentes. Entretanto, eles não relatam a identificação dos pesos atribuídos aos fatores de risco nem o emprego de técnicas estatísticas adequadas. As únicas escalas com valor preditivo testado foram as de Norton, Waterlow e Braden ${ }^{(2)}$.

A literatura sustenta que a avaliação de risco deve sempre estar acompanhada do julgamento clínico do enfermeiro, mas se este for aplicado isoladamente, com exceção do julgamento de enfermeiros estomaterapeutas padrão ouro, será menos eficaz do que a aplicação dos instrumentos de avaliação ${ }^{(3-6)}$.

A European Pressure Ulcer Advisory Panel (EPUAP) órgão que se originou em Londres, em 1996, que, periodicamente, se reúne e apresenta diretrizes de prevenção e tratamento baseadas em evidências, recomenda que um instrumento de avaliação de risco deve incluir: condição geral e avaliação da pele, mobilidade, umidade, incontinência, nutrição e dor (7). Das escalas mais utilizadas no mundo, Norton, Braden e Waterlow, a única que apresenta a avaliação da pele, como fator de risco, é a de Waterlow ${ }^{(8)}$.

A escala de Waterlow foi baseada na de Norton, porém, com avaliação de um maior número de itens, que foram obtidos após longa investigação dos fatores etiológicos das $\mathrm{UP}^{(9)}$.

A patogênese das úlceras por pressão é um problema complexo e três fatores principais são de grande importância nesse processo: a pressão direta, as forças de cisalhamento e fricção. Um paciente que sofre uma combinação de fatores predisponentes é mais suscetível ao desenvolvimento de úlcera. Tais fatores podem ser divididos em dois grupos: Intrínsecos, são os aspectos do tipo de compleição corporal do paciente, mobilidade, estado nutricional, incontinência, infecção e condições clínicas; por exemplo, doenças malignas, neurológicas e anemia; Fatores extrínsecos, incluem os efeitos externos de drogas, distribuição de peso, regime de tratamento (clínico ou cirúrgico, cuidados intensivos), cuidados de higiene e técnicas de manuseio do paciente ${ }^{(10)}$.

Desta forma, este estudo teve como objetivo aplicar clinicamente a escala de Waterlow, já adaptada à língua portuguesa ${ }^{(11)}$, a fim de conhecer os índices de sensibilidade e especificidade para predizer o desenvolvimento de úlceras por pressão.

\section{MÉTODOS}

Trata-se de um estudo analítico, descritivo e prospectivo realizado em um Hospital Universitário de porte extra localizado no Município de São Paulo, nas unidades de Ortopedia, Clínica Médica e Retaguarda do Pronto-Socorro. O projeto de pesquisa foi previamente aprovado pelo Comitê de Ética em Pesquisa da Universidade Federal de São Paulo. Estas unidades foram escolhidas por apresentarem pacientes clinicamente suscetíveis ao desenvolvimento de úlceras por pressão, em razão da cronicidade das doenças, da gravidade, da média elevada do tempo de internação e, sobretudo, pelo elevado grau de dependência dos mesmos.

Nestas unidades a sistematização da assistência de enfermagem era realizada e o enfermeiro avaliava os riscos para desenvolvimento de úlceras por pressão, implementava as intervenções necessárias para prevenção de forma empírica, não sendo, portanto, utilizado nenhum instrumento padronizado. $\mathrm{Na}$ época do estudo, os materiais padronizados no hospital para prevenção consistiam em colchão de espuma piramidal e placas de substância hidrocolóide que eram aplicadas nas áreas consideradas mais suscetíveis ao desenvolvimento de úlceras por pressão. A mudança de decúbito a cada duas horas fazia parte da prescrição de enfermagem de todos os pacientes acamados.

Os critérios de inclusão adotados foram: ser adulto ( $\geq 18$ anos), não apresentar úlcera por pressão no momento da coleta de dados e ter perspectiva de tempo de internação igual ou superior a 15 dias.

\section{Coleta de Dados}

Para a coleta de dados, foi desenvolvido um instrumento exclusivo que continha a versão traduzida e 
adaptada da escala (anexo), dados de identificação do paciente e dados demográficos.

A avaliação foi realizada uma vez ao dia durante 15 dias consecutivos em todos os pacientes pela própria pesquisadora especialista em UTI e capacitada para utilização dos instrumentos de avaliação de risco por meio de prática clínica, a fim de identificar o aparecimento de lesões nesse período, após obtenção da assinatura do Termo de Consentimento Livre e Esclarecido. A coleta dos dados foi realizada em 15 dias e fundamentou-se em estudo anterior que demonstrou que o período crítico de desenvolvimento de lesões por pressão era de até 14 dias, após a admissão( ${ }^{(8)}$.

Os pacientes que desenvolveram UP, tiveram registrados o dia de aparecimento, o local e o estágio da úlcera e continuaram sendo avaliados até o final dos 15 dias para identificação de novas lesões e acompanhamento do desfecho de sua internação (alta, óbito ou transferência) para fins estatísticos.

O estadiamento das úlceras foi classificado conforme as diretrizes de prevenção e tratamento da $\operatorname{EPUAP}^{(7)}$.

Ao final da coleta de dados, todos os pacientes que atenderam aos critérios de inclusão tiveram 15 avaliações no total.

\section{Análise estatística}

A associação entre a variável presença de úlcera por pressão e as demais variáveis qualitativas foram avaliadas pelo teste do Qui-quadrado. A comparação, entre os grupos com e sem úlcera por pressão em relação aos escores, foi feita pela prova não-paramétrica MannWhitney para amostras independentes, em razão da natureza das variáveis, e também, dentro de cada grupo os valores do escore total foram comparados nas 15 avaliações pela prova não-paramétrica de Friedman, a fim de encontrar diferença estatística entre os escores dos pacientes dos grupos com e sem lesão.

A análise de regressão logística com o método Stepwise para seleção e análise do grau de importância de variáveis foi aplicada entre os fatores contemplados na escala, para encontrar, quais tiveram maior associação com a ocorrência de úlceras por pressão.

\section{Escala de Waterlow}

O objetivo da escala de Waterlow é criar consciência sobre os fatores causais e oferecer um método de avaliação de risco, grau de lesão e prevenção ou tratamento ativo necessário. A avaliação do paciente contém sete tópicos principais: relação peso/altura (IMC), avaliação visual da pele em áreas de risco, sexo/ idade, continência, mobilidade, apetite, e medicações. além de quatro itens que pontuam fatores de risco especiais, subnutrição do tecido celular, déficit neurológico, tempo de cirurgia, acima de duas horas e trauma abaixo da medula lombar. Quanto mais alto o escore, maior será o risco de desenvolver a lesão. Os pacientes são estratificados em três grupos, conforme a pontuação: em risco (escore de 10 a 14); alto risco (escore de 15 a 19) e altíssimo risco de desenvolvimento de UP (escore $\geq 20$ ). Se o paciente entrar em uma categoria de risco, então será possível acessar uma lista de sugestões de medidas preventivas ${ }^{(10)}$.

\section{RESULTADOS}

Durante o período de coleta de dados, setembro a novembro de 2001, 44 pacientes atenderam aos critérios de inclusão previamente mencionados, 22 (50\%) eram do sexo feminino e $22(50 \%)$ do sexo masculino. Vinte três pacientes $(52,3 \%)$ desenvolveram úlceras por pressão, a maioria destes $(78 \%)$ encontrava-se internado na unidade de retaguarda do Pronto Socorro.

A idade dos pacientes variou de 18 a 86 anos $(54 \pm 20$ anos). Dos pacientes que desenvolveram UP, 45\% tinham idade igual ou superior a 65 anos e, pouco mais de $60 \%$ dos pacientes sem a lesão apresentavam idade não superior a 49 anos $(p=0,020)$.

Quanto à localização das úlceras, foram encontradas $82 \%$ das lesões na região sacra e 18\% em calcâneos, sendo a maioria em estágios I e II (43\% e 25\%, respectivamente), segundo a classificação da EPUAP (7). As úlceras apareceram entre os $2^{\circ}$ e $15^{\circ}$ dias $(8 \pm 5$ dias $)$; nos cinco pacientes que apresentaram uma segunda lesão, ela se desenvolveu entre os $13^{\circ}$ e $15^{\circ}$ dias de avaliação.

Uma elevada diversidade de diagnósticos médicos ( $\mathrm{n}=$ 55) foi encontrada e a maior parte dos pacientes possuía mais de um diagnóstico, por esse motivo não foi submetido a nenhum teste estatístico. O diagnóstico mais freqüente foi neoplasia (26\%), seguido de doenças neurológicas e pulmonares (16\% cada).

De todos os itens avaliados na escala de Waterlow, seis deles foram estatisticamente significantes na população que desenvolveu UP: tipo de pele classificada como não saudável; sexo feminino, idade superior a 50 anos, incontinência fecal, restrição ao leito ou inércia, e alteração do apetite (Tabela 1$)$.

$\mathrm{Na}$ análise de regressão logística, demonstrou-se que a equação do modelo final formada por uma constante e pelos escores de tipo de pele e apetite foram fatores preditores significantes de ocorrência de UP (Tabela 2).

Quando comparados os valores do escore total nas 15 avaliações (Tabela 3) foi encontrada diferença estatisticamente significante entre os pacientes com e sem úlcera por pressão quanto ao escore total da escala de Waterlow nas 15 aplicações da escala ( $\mathrm{p}<0,001)$. O grupo sem úlcera apresentou escore total significantemente menor 
do que o grupo com úlcera. A comparação entre os escores nas 15 aplicações dentro de cada grupo mostrou que a variação encontrada entre as aplicações não se mostrou estatisticamente significante nos dois grupos $(\mathrm{p}$ $=0,435$ para o grupo sem úlcera e $\mathrm{p}=0,751$ para o grupo com úlcera).

Tabela 1 - Distribuição dos pacientes que desenvolveram UP, segundo os itens da Escala de Waterlow com significância estatística n população de estudo. São Paulo, 2006.

\begin{tabular}{lccc}
\hline $\begin{array}{l}\text { Itens avaliados na } \\
\text { escala de Waterlow }\end{array}$ & $\begin{array}{c}\text { Pacientes } \\
\text { sem UP } \\
(21)\end{array}$ & $\begin{array}{c}\text { Pacientes } \\
\text { com UP } \\
\text { N }(\%)\end{array}$ & $p^{*}$ \\
\hline $\begin{array}{l}\text { Tipo de Pele } \\
\text { Não saudável }\end{array}$ & $3(6)$ & \\
$\begin{array}{l}\text { Sexo feminino } \\
\text { Idade (50 - 80 anos) }\end{array}$ & $7(33)$ & $15(65)$ & 0,003 \\
$\begin{array}{l}\text { Continência } \\
\text { (incontinência fecal) }\end{array}$ & $3(14)$ & $16(69)$ & 0,020 \\
$\begin{array}{l}\text { Mobilidade } \\
\text { (Restrito / Contido }\end{array}$ & & $19(83)$ & $<0,001$ \\
$\begin{array}{l}\text { ao leito) } \\
\text { Apetite (abaixo do } \\
\text { normal/ somente }\end{array}$ & $7(33)$ & & \\
$\begin{array}{l}\text { líquidos/ SNE para } \\
\text { gavagem / }\end{array}$ & & & \\
anorético) & $8(38)$ & $20(87)$ & 0,001 \\
\hline
\end{tabular}

$*$ Prova de Mann-Whitney

Segundo a classificação de risco da escala de Waterlow, encontrou-se diferença estatisticamente significante entre os pacientes com e sem úlcera por pressão. $\mathrm{Na}$ avaliação dos escores no primeiro dia de aplicação da escala, $87 \%$ dos pacientes que desenvolveram a lesão foram classificados em alto ou altíssimo risco (43,5\% para cada grupo) e dos pacientes sem UP $33,3 \%$ foram classificados sem risco e $52,4 \%$ em risco (Figura 1).

Diante dos resultados de igualdade entre os escores totais no decorrer das 15 aplicações da escala, foram escolhidos os escores totais das últimas aplicações da escala ao grupo sem úlcera e da aplicação anterior ao dia do aparecimento da lesão ao grupo com úlcera, para a previsão de um ponto de corte para o escore total da escala de Waterlow (Figura 2). Diferença estatisticamente significante foi verificada entre os dois grupos em relação ao escore total da escala $(18,1 \pm 3,4$ vs.12,8 45,4 ; $p<0,001)$. O escore 15 demonstrou ser o melhor para predizer o desenvolvimento de úlceras por pressão, com sensibilidade e especificidade de $87 \%$ e $76 \%$, respectivamente.

Tabela 3 - Medidas descritivas do escore total da escala de Waterlow dos pacientes sem e com UP nas 15 aplicações e as respectivas comparações. São Paulo, 2003.

\begin{tabular}{|c|c|c|c|c|c|}
\hline \multirow{2}{*}{$\begin{array}{c}\text { Aplicação } \\
\text { da escala }\end{array}$} & \multicolumn{2}{|c|}{ Sem UP } & \multicolumn{2}{|c|}{ Com UP } & \multirow{2}{*}{$\begin{array}{c}\text { Prova de } \\
\text { Mann- } \\
\text { Whitney }\end{array}$} \\
\hline & $\begin{array}{l}\text { Média } \\
\text { d.p. }\end{array}$ & $\begin{array}{c}\text { Mediana } \\
\text { Min./ } \\
\text { Max. }\end{array}$ & $\begin{array}{l}\text { Média } \\
\text { d.p. }\end{array}$ & $\begin{array}{c}\text { Mediana } \\
\text { Min./ } \\
\text { Max. }\end{array}$ & \\
\hline \multirow{2}{*}{$1^{a}$} & 10,8 & 10 & 18,2 & 18 & $\mathrm{p}<0,001$ \\
\hline & 5,2 & $4 / 26$ & 3,7 & $11 / 24$ & * \\
\hline \multirow{2}{*}{$2^{a}$} & 11,0 & 10 & 18,2 & 18 & $\mathrm{p}<0,001$ \\
\hline & 5,3 & $4 / 26$ & 3,7 & $11 / 24$ & * \\
\hline \multirow{2}{*}{$3^{a}$} & 11,0 & 10 & 18,2 & 18 & $\mathrm{p}<0,001$ \\
\hline & 5,2 & $4 / 26$ & 3,7 & $11 / 24$ & * \\
\hline \multirow{2}{*}{$4^{a}$} & 11,0 & 10 & 18,2 & 18 & $\mathrm{p}<0,001$ \\
\hline & 5,2 & $4 / 26$ & 3,7 & $11 / 24$ & * \\
\hline \multirow{2}{*}{$5^{a}$} & 11,2 & 10 & 18,2 & 18 & $\mathrm{p}<0,001$ \\
\hline & 5,5 & $4 / 26$ & 3,7 & $11 / 24$ & $*$ \\
\hline \multirow{2}{*}{$6^{a}$} & 11,5 & 11 & 18,2 & 18 & $\mathrm{p}<0,001$ \\
\hline & 5,7 & $4 / 27$ & 3,7 & $11 / 24$ & $*$ \\
\hline \multirow{2}{*}{$7^{a}$} & 11,7 & 11 & 18,3 & 19 & $\mathrm{p}<0,001$ \\
\hline & 5,4 & $4 / 26$ & 3,6 & $11 / 24$ & $*$ \\
\hline \multirow{2}{*}{$8^{\mathrm{a}}$} & 11,7 & 11 & 18,3 & 19 & $\mathrm{p}<0,001$ \\
\hline & 5,4 & $4 / 26$ & 3,6 & $11 / 24$ & $*$ \\
\hline \multirow{2}{*}{$9^{a}$} & 11,7 & 11 & 18,3 & 19 & $\mathrm{p}<0,001$ \\
\hline & 5,4 & $4 / 26$ & 3,6 & $11 / 24$ & $*$ \\
\hline \multirow{2}{*}{$10^{\mathrm{a}}$} & 11,7 & 11 & 18,2 & 19 & $\mathrm{p}<0,001$ \\
\hline & 5,4 & $4 / 26$ & 3,6 & $11 / 24$ & $*$ \\
\hline \multirow{2}{*}{$11^{\mathrm{a}}$} & 11,7 & 11 & 18,3 & 19 & $\mathrm{p}<0,001$ \\
\hline & 5,4 & $4 / 26$ & 3,8 & $11 / 26$ & * \\
\hline \multirow[b]{2}{*}{$12^{\mathrm{a}}$} & 11,5 & 11 & 18,3 & 19 & $\mathrm{p}<0,001$ \\
\hline & 5,0 & $4 / 23$ & 3,8 & $11 / 26$ & * \\
\hline \multirow{2}{*}{$13^{\mathrm{a}}$} & 11,5 & 11 & 18,3 & 19 & $\mathrm{p}<0,001$ \\
\hline & 5,0 & $4 / 23$ & 3,8 & $11 / 26$ & $*$ \\
\hline \multirow{2}{*}{$14^{\mathrm{a}}$} & 11,5 & 11 & 18,3 & 19 & $\mathrm{p}<0,001$ \\
\hline & 5,0 & $4 / 23$ & 3,8 & $11 / 26$ & F $\quad *$ \\
\hline \multirow{2}{*}{$15^{a}$} & 11,5 & 11 & 18,3 & 19 & $\mathrm{p}<0,001$ \\
\hline & 5,0 & $4 / 23$ & 3,8 & $11 / 26$ & * \\
\hline $\begin{array}{l}\text { Prova de } \\
\text { Friedman }\end{array}$ & \multicolumn{2}{|c|}{$\mathrm{p}=0,435$} & \multicolumn{2}{|c|}{$\mathrm{p}=0,751$} & \\
\hline
\end{tabular}

Tabela 2 - Resultados da regressão logística pelo método de Stepwise dos fatores tipo de pele e apetite. São Paulo, 2003.

\begin{tabular}{lccccc}
\hline \multicolumn{1}{c}{ Variável } & Coeficiente $(\beta)$ & Erro padrão $\beta$ & $\mathrm{p}$ & $\mathrm{OR}$ & IC $_{95 \%}$ OR \\
\hline Tipo de pele & 1,4091 & 0,5936 & $0,018 *$ & 4,0924 & {$[1,2786 ; 13,0988]$} \\
Apetite & 1,6285 & 0,4992 & $0,001 *$ & 5,0964 & {$[1,9159 ; 13,5565]$} \\
Constante & $-3,3804$ & 1,1079 & $0,002 *$ & --- & --- \\
\hline
\end{tabular}

Teste de ajuste do modelo: $\mathrm{p}<0,001 *$ 


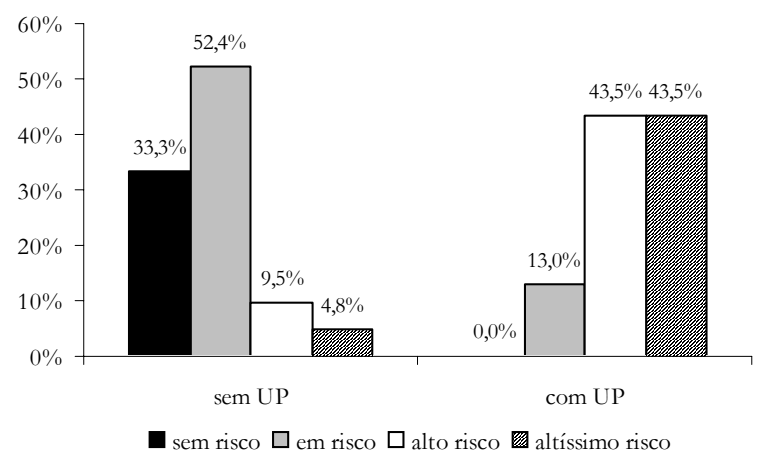

Figura 1 - Distribuição dos pacientes com e sem UP, segundo a classificação de risco da escala de Waterlow na primeira aplicação.

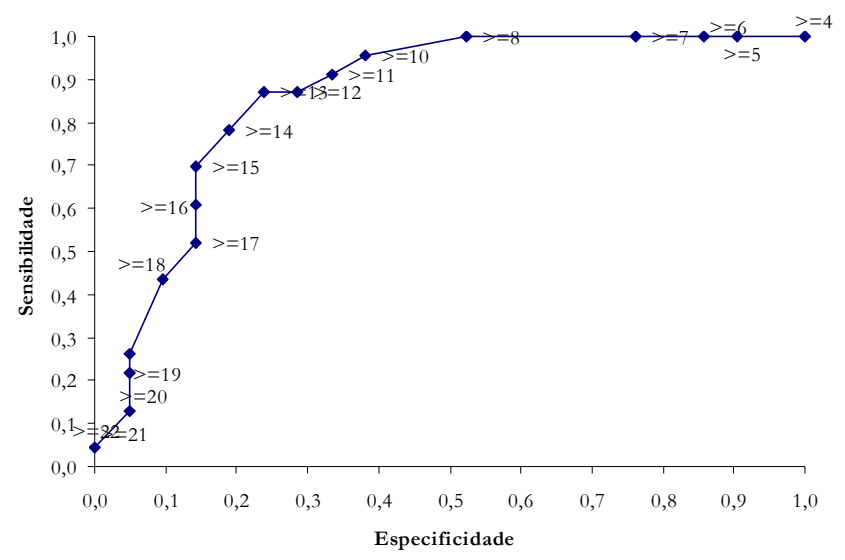

Figura 2 - Gráfico demonstrativo da curva ROC para o escore total da $15^{\mathrm{a}}$ aplicação aos pacientes sem UP e da aplicação do dia anterior ao aparecimento da lesão aos pacientes com UP.

\section{DISCUSSÃO}

A escala de Waterlow adaptada à língua portuguesa demonstrou ser um instrumento eficaz para predizer o desenvolvimento de úlceras por pressão na população do estudo, quando foram selecionados os pacientes que estavam em risco para desenvolvimento destas lesões.

A análise de confiabilidade da escala adaptada demonstrou que os escores para tipo de pele e apetite foram os mais significantes para predizer o desenvolvimento das úlceras por pressão na população do estudo. A interpretação dos dados mostrou que o acréscimo de um ponto no escore para apetite acarreta um aumento de cinco vezes na probabilidade de ocorrer úlcera por pressão e o acréscimo de um ponto no escore para tipo de pele provoca um aumento de quatro vezes em ocorrer a lesão.

A avaliação do tipo de pele apresenta extrema importância na caracterização do risco de desenvolvimento de UP. Em um estudo em que foram comparadas três escalas de avaliação de risco, encontrou- se que $86 \%$ dos pacientes que desenvolveram UP, apresentavam pele não saudável, ou seja, muito fina, seca, com edema, úmida e pegajosa (em altas temperaturas), descorada, quebradiça ou marcada ${ }^{(8)}$, valores estes semelhantes aos apresentados neste estudo (96\%).

Em estudos nos quais a escala de Waterlow foi testada clinicamente, o item apetite aparece relacionado ao estado nutricional do paciente, ou seja, comparando a quantidade e o modo como o paciente se alimenta e seu estado nutricional ${ }^{(2,8)}$. Alguns desses estudos demonstraram que os pacientes em estado nutricional abaixo do normal apresentam maior risco de desenvolvimento de $\mathrm{UP}^{(12-14)}$, assim, deve ser realizado um acompanhamento nutricional adequado nos pacientes em risco ${ }^{(13-14)}$. Em nosso estudo, este item foi significante para predizer o desenvolvimento de UP. Dos pacientes que não desenvolveram a lesão, a maioria não tinha alterações no padrão de alimentação, já para os pacientes que desenvolveram a úlcera, apenas três $(13 \%)$ não apresentaram alterações no apetite.

Para o desenvolvimento da úlcera por pressão na população do estudo, outros fatores foram relevantes em relação ao sexo e desenvolvimento de UP. Encontrou-se em alguns estudos uma incidência maior No sexo feminino de até $77 \%{ }^{(3,14-15)}$, valores estes semelhantes aos obtidos no presente estudo $(65 \%)$.

$\mathrm{Na}$ escala de Waterlow, quanto maior a idade do paciente maior será a pontuação, verificou-se neste estudo em quase metade dos pacientes que desenvolveram a lesão, idade acima dos 65 anos. A literatura demonstra que esta idade, quando associada a um ou mais fatores de risco provoca um aumento significativo na probabilidade de desenvolvimento de $\mathrm{UP}^{(12-13,15)}$ e também associa a úlcera por pressão como uma complicação importante para predizer a mortalidade de pacientes idosos ${ }^{(16)}$.

O item continência, em especial a incontinência fecal, demonstrou ser um dos mais relevantes para o desenvolvimento de úlcera por pressão, chegando a estar relacionado a um aumento de cerca de 22 vezes o risco de desenvolvimento de UP quando associado à incontinência urinária ${ }^{(17)}$. O cuidado com a pele das regiões próximas às áreas de incontinência deveria ser padronizado como medida preventiva, por exemplo, com o uso de cremes como barreira de proteção dessas áreas; já que a incontinência é um fator de risco potencial para prejuízo da integridade da pele. Sendo assim, além da escala de Waterlow, este item está incluso em diversas escalas de avaliação de risco para UP e, comprovadamente, tem um alto poder preditivo para esta lesão, inclusive quando analisado isoladamente ${ }^{(7,10)}$.

Como mencionado anteriormente, os fatores mais importantes para desenvolvimento da UP são: a pressão direta e as forças de cisalhamento e fricção, fatores estes presentes em pacientes com algum grau de imobilidade no leito ou em cadeira. Nos estudos que tratam das 
complicações médicas em portadores de lesões medulares observou-se que a úlcera por pressão foi uma das mais freqüentes, embora o uso de medidas preventivas simples por parte da equipe de enfermagem, como, por exemplo, a realização de mudanças de decúbito e mobilizações freqüentes no leito tenham diminuído sensivelmente a incidência da lesão nesses $\operatorname{casos}^{(10,14)}$.

Neste estudo, não foi usada a lista de medidas preventivas contidas na versão original da escala de Waterlow $^{(9)}$, pois não fazia parte dos objetivos alterar as rotinas das unidades quanto à prevenção e tratamento das úlceras por pressão e, como citado anteriormente, nesta rotina, inclui-se a mudança de decúbito dos pacientes, de acordo com o julgamento clínico do enfermeiro da unidade.

Ao analisar as áreas atingidas pela lesão, foram encontradas apenas lesões em calcâneos $(18 \%)$ e região sacra $(82 \%)$. Esta última é considerada uma das mais suscetíveis para desenvolvimento de ulceras por pressão em razão das proeminências ósseas e proximidade com áreas de incontinência ${ }^{(12,15)}$ e, por isto deve ser constantemente avaliada. Em relação ao tipo de pele, em quase todos os pacientes que desenvolveram a lesão, verificou-se que a pele não era saudável. A literatura aponta que, além da região sacra, altamente suscetível, devem ser observadas também as regiões do quadril, calcâneos, tornozelos, cotovelos e occipto, a fim de identificar precocemente sinais desse tipo de lesão e condições de risco, como pele seca, quebradiça, com eritema, maceração, fragilidade, temperatura elevada ou endurecimento $^{(7)}$.

Neste estudo, as lesões estavam em grande parte no estágio I (43\%), que é definido como um eritema não branqueável na pele intacta ${ }^{(7)}$. A literatura demonstra que a prevalência de úlceras por pressão no estágio I é alta ${ }^{(4,8,12)}$. No entanto, esta fase é de difícil identificação, talvez pelo fato de não apresentar lesões efetivas consideradas como pré-úlcera $^{(18)}$, ou não fazendo parte da casuística em estudos sobre incidência em razão da possível subnotificação desta fase da úlcera por pressão ${ }^{(10)}$.

Em geral, os estudos para avaliação de risco de úlceras por pressão são realizados pautados em uma clientela específica; em sua maioria, nas unidades de terapia intensiva, em pacientes geriátricos e portadores de lesões medulares, cada um com fatores de risco específicos ${ }^{(2-4)}$. Isto pode explicar os resultados contrastantes apresentados no valor preditivo das diversas escalas de avaliação de risco. Neste estudo, pela análise da curva ROC, o escore 15 foi encontrado sendo o que apresentou valores mais adequados de sensibilidade e especificidade, $87 \%$ e $76 \%$, respectivamente. Em estudos clínicos, o escore 16 é apontado como ponto de corte recomendado para pacientes em risco ${ }^{(8-9)}$.

Em um estudo prospectivo, realizado na Holanda, sobre a rotina do uso de escalas de avaliação de risco para predizer úlceras por pressão em pacientes neurológicos, geriátricos e de clínica médico-cirúrgica, foram avaliadas as escalas de Norton, Waterlow e Braden, usando os escores médios recomendados, sendo encontrados valores preditivos positivos bastante baixos para todas escalas, $7 \%$, 5,3\% e 7,8\%, respectivamente. Assim como os valores de sensibilidade e especificidade, tanto para a escala de Braden, 43,5\% e 67.8\%, como Norton 46,2\% e 60,4\% e Waterlow 89,5\% e 22,4\%, respectivamente ${ }^{(2)}$.

Cabe ressaltar que as escalas de avaliação de risco devem ser aplicadas o mais precocemente possível e, de preferência, na admissão do paciente, podendo, inclusive, estar impressa no histórico de enfermagem, o que agilizaria a implementação das medidas preventivas adequadas, reduzindo a incidência destas lesões ${ }^{(7,10)}$. Um programa de prevenção e tratamento deve ser adotado, para assegurar que todos os pacientes potencialmente suscetíveis, de acordo com o julgamento clínico do enfermeiro, sejam avaliados a partir da admissão e em intervalos apropriados. Desse modo, dependendo de seu escore de risco, ter uma lista de medidas preventivas disponíveis que permita que estes cuidados sejam dispostos onde houver maior necessidade; evoluir a efetividade das medidas preventivas pela reavaliação do paciente quanto às áreas de pressão; reciclar a equipe quanto ao uso destas medidas e avaliar as necessidades de enfermagem na unidade, para aperfeiçoar os cuidados $^{(10,14)}$.

\section{CONCLUSÃO}

A avaliação de risco é uma ferramenta eficaz na prevenção das úlceras por pressão e a literatura ressalta a importância desse tipo de instrumento para nortear as ações de enfermagem, no que diz respeito ao uso adequado e racional das medidas preventivas, para redução do tempo de internação hospitalar, dos custos com o tratamento e, sobretudo, diminuir o sofrimento e dor dos pacientes, melhorando a qualidade do cuidado de enfermagem. $\mathrm{O}$ uso desses instrumentos deve sempre estar associado ao julgamento clínico do enfermeiro, para norteá-lo na implementação de ações de enfermagem condizentes com o risco de cada paciente.

Foi possível concluir que, diferente de outras investigações de aplicação clínica da escala de Waterlow, que estabeleciam o escore 16, como ponto de corte para detectar pacientes em risco, neste estudo foi encontrado o escore 15 como o melhor para predizer o desenvolvimento de úlceras por pressão que apresentou os melhores índices de sensibilidade e especificidade, $87 \%$ e $76 \%$, respectivamente.

Embora esta pesquisa apresente algumas limitações, 
como o número reduzido de pacientes e a diversificação no que diz respeito à unidade de internação e ao diagnóstico inicial, foi capaz de demonstrar a aplicabilidade da escala de Waterlow adaptada em pacientes considerados graves e não graves, com diferentes fatores de risco para desenvolvimento de úlcera por pressão, internados fora da unidade de terapia intensiva.

Desta forma, utilizando a escala de Waterlow adaptada à língua portuguesa apresentada, outros estudos clínicos poderão ser realizados em nosso meio, a fim de confirmar a aplicabilidade e a utilidade da mesma em diferentes populações.

\section{REFERÊNCIAS}

1. Goldstone LA, Goldstone J. The Norton score: an early warning of pressure sores? J Adv Nurs. 1982; 7(5):419-26.

2. Schoonhoven L, Haalboom JR, Bousema MT, Algra A, Grobbee DE, Grypdonck MH, Buskens E; prePURSE study group. The prevention and pressure ulcer risk score evaluation study. Prospective cohort study of routine use of risk assessment scales for prediction of pressure ulcers. BMJ. 2002; 325(7368):797.

3. Defloor T, Grypdonck MF. Pressure ulcers: validation of two risk assessment scales. J Clin Nurs. 2005; 14(3):373-82.

4. Kwong E, Pang S, Wong T, Ho J, Shao-ling X, Li-jun T. Predicting pressure ulcer risk with the modified Braden, Braden, and Norton scales in acute care hospitals in Mainland China. Appl Nurs Res. 2005; 18(2):122-8.

5. Brown SJ. The Braden Scale. A review of the research evidence. Orthop Nurs. 2004; 23(1):30-8.

6. Thompson D. An evaluation of the Waterlow pressure ulcer risk-assessment tool. Br J Nurs. 2005; 14(8):455-9.

7. EPUAP [homepage on the Internet]. Oxford: European Pressure Ulcer Advisory Panel. Pressure Ulcer Prevention and Treatment Guidelines: c1998 - [updated 2006 Sep 29; cited 2006 Oct 10]. Available from: URL: http:// www.epuap.org/gltreatment.html

8. Pang SM, Wong TK. Predicting pressure sore risk with the Norton, Braden, and Waterlow scales in a Hong Kong rehabilitation hospital. Nurs Res. 1998; 47(3):147-53.

9. Waterlow J. Pressure sores: a risk assessment card. Nurs Times. 1985; 81(48):49-55.

10. Waterlow J. Tissue viability. Prevention is cheaper than cure. Nurs Times. 1988; 84 (25):69-70.

11. Rocha ABL. Tradução para a língua portuguesa, adaptação transcultural e aplicação clínica da escala de Waterlow para avaliação de risco de desenvolvimento de úlcera de decúbito [tese]. São Paulo: Universidade Federal de São Paulo. Escola Paulista de Medicina; 2003.

12. Rogenski NMB, Santos VLCG. Estudo sobre a incidência de úlceras por pressão em um hospital universitário. Rev Latinoam Enfermagem. 2005; 13(4):474-80.

13. Casimiro C, Garcia-de-Lorenzo A, Usan L. Prevalence of decubitus ulcer and associated risk factors in an institutionalized Spanish elderly population. Nutrition. 2002; 18(5):408-14.

14. Lindgren M, Unosson M, Krantz AM, Ek AC. Pressure ulcer risk factors in patients undergoing surgery. J Adv Nurs. 2005; 50(6):605-12.

15. Berlowitz DR, Bezerra HQ, Brandeis GH, Kader B, Anderson JJ. Are we improving the quality of nursing home care: the case of pressure ulcers. J Am Geriatr Soc. 2000; 48 (1):59-62.

16. Siqueira AB, Cordeiro RC, Perracini MR, Ramos LR. Impacto funcional da internação hospitalar de pacientes idosos. Rev Saúde Pública = J Public Health. 2004; 38(5): 687-94.

17. Maklebust J. Interrupting the pressure ulcer cycle. Nurs Clin North Am. 1999; 34(4):861-71, vi.

18. Blanes L, Duarte IS, Calil JA, Ferreira LM. Avaliação clínica e epidemiológica das úlceras por pressão em pacientes internados no Hospital São Paulo. Rev Assoc Med Bras. 2004; 50 (2):182-7. 
ANEXO

ESCALA DE WATERLOW ADAPTADA À LÍNGUA PORTUGUESA

\begin{tabular}{|c|c|c|c|}
\hline Itens & Pontos & Itens & Pontos \\
\hline IMC & & \multicolumn{2}{|c|}{ SUBNUTRIÇÃO DO TECIDO CELULAR } \\
\hline Média & 0 & Caquexia & 8 \\
\hline Acima da média & 1 & Insuficiência Cardíaca & 5 \\
\hline Obeso & 2 & Doença Vascular Periférica & 5 \\
\hline Abaixo da média & 3 & Anemia & 2 \\
\hline TIPO DE PELE (em áreas de risco) & & Fumante & 1 \\
\hline Saudável & 0 & DEFICIÊNCIA NEUROI & \\
\hline Muito fina & 1 & Diabetes & 4 \\
\hline Seca & 1 & Paraplegia Motora ou Sensi & 6 \\
\hline Com edema & 1 & \multicolumn{2}{|c|}{ CIRURGIA GRANDE PORTE/ TRAUMA } \\
\hline Úmida e pegajosa (em alta temperatura) & 1 & Abaixo da medula lombar & 5 \\
\hline Descorada & 2 & Acima de duas horas & 5 \\
\hline Quebradiça / marcada & 3 & APETITE & \\
\hline SEXO / IDADE & & Normal & 0 \\
\hline Masculino & 1 & Pouco & 1 \\
\hline Feminino & 2 & Somente líquido & 2 \\
\hline $14-49$ & 1 & SNG/E & \\
\hline $50-64$ & 2 & Anorético & 3 \\
\hline $65-74$ & 3 & MEDICAÇÃO & \\
\hline $75-80$ & 4 & Alta dosagem: & 4 \\
\hline $80+$ & 5 & Esteróides & \\
\hline CONTINENCIA & & Citotóxicos & \\
\hline Uso de SVD ou continente & 0 & Antiinflamatório & \\
\hline Ocasionalmente incontinente & 1 & & \\
\hline Uso de SVD e incontinente fecal & 2 & & \\
\hline Duplamente incontinente & 3 & & \\
\hline MOBILIDADE & & & \\
\hline Total & 0 & & \\
\hline Inquieto / Agitado & 1 & & \\
\hline Apático & 2 & TOTAL & \\
\hline Restrito / Contido & 3 & EM RISCO & $10+$ \\
\hline Inerte & 4 & ALTO RISCO & $15+$ \\
\hline Dependente de cadeira de rodas & 5 & ALTÍSSIMO RISCO & $20+$ \\
\hline
\end{tabular}

Adaptado de: Rocha ABL. Tradução para a língua portuguesa, adaptação transcultural e aplicação clínica da escala de Waterlow para avaliação de risco de desenvolvimento de úlcera de decúbito [tese]. São Paulo, Universidade Federal de São Paulo Departamento de Enfermagem; 2003. 